\title{
Multi-criteria Identification of VibroBot Dynamic Characteristics
}

\author{
Ivan A. Loukanov ${ }^{1}$, Venko G. Vitliemov ${ }^{2}$, Ivelin V. Ivanov ${ }^{2}$ \\ ${ }^{1}$ Department of Mechanical Engineering, University of Botswana, Gaborone, Botswana \\ ${ }^{2}$ Department of Engineering Mechanics, University of Ruse, Ruse, Bulgaria
}

\begin{abstract}
A multi-criteria parametric identification problem of elastic and dissipative characteristics is formulated and solved using the measured acceleration of free damped oscillations of a prototype, which is a mechanical system with vibration propulsion (VibroBot). The oscillating process is simulated by single-mass mechanical and mathematical models having four different dynamic characteristics. Five criteria for correlation between the simulated and the measured values of the acceleration and their extreme values along with the instants at which they occurred, are chosen. The problem of vector identification of the elasticity and the viscous coefficients along with the dry friction is formulated. The problem is solved by means of two-stage procedure based on the Parameter Space Investigation (PSI) method for studying multidimensional space through pseudouniform exploration. A Pareto optimal set with compromised solutions is determined. By using the PSI method and employing a technique known as , $\mu$-selection“, ranked Pareto subsets are separated. The choice of simulation model corresponding to the Salukvadze optimum solution of highest rank is validated.
\end{abstract}

Key words: $\mu$-selection, Pareto optimal set, PSI method, Salukvadze optimum, VibroBot.

\section{INTRODUCTION}

In the modern self-propelled mobile systems a valuable class of mechanical systems with inertial propulsion oriented towards non-traditional applications is emerged [1], [2]. The mobile robots with vibration propulsion known as Vibrobots are objects of great interest inspired by their advantages when operating at unusual conditions, the necessity of being miniature, to comply with the ecology requirements and to be energy efficient [3], [4], [5]. Their distinguished features are that they operate in the vicinity of the main resonance and dynamic nonlinearity, which makes their studies challenging. Creating an adequate simulating model of a real VibroBot is associated with determination and characterization of its dynamic forces.

In the present study the problem of multi-criteria identification of dynamic characteristics of the vibration-driven VibroBot by using its experimentally determined acceleration of free damped vibrations is formulated and solved.

\section{DETERMINATION OF THE EXPERIMENTL DATA}

In Fig. 1, the prototype of a functional VibroBot investigated in [6] and [7] is presented. In this model the counter rotation of the unbalanced masses is transformed into a unidirectional non-uniform pulsating motion of the mechanical system. The unidirectional motion of the VibroBot is achieved by means of one-way rotating bearings build into the hubs of all wheels. These bearings allow rotation of the wheels during a forward motion of the VibroBot and block the rotation of the wheels in the opposite motion.

In order to determine the generalized elastic and dissipative characteristics of the prototype model, numerous experiments were conducted when the chassis of the Vibrobot is fixed and kept stationary during the experiments as seen in Fig. 2. The free damped oscillations of the propulsion mechanism (shaker) are excited by displacing the mechanism from equilibrium position to an amplitude value and freeing it from rest. Then the shaker starts performing free oscillations until they vanish. The signal of the accelerometer attached to the propulsion mechanism is recorded for about four seconds and continuously saved by the data log and measuring portable system - LabQuest $2^{\mathrm{TM}}$ as this is shown in the experimental investigation [7]. Then the recorded signal of the acceleration is further subjected to smoothening as per the procedure explained in the following sections of this study in order to determine precisely the elastic and dissipative characteristics of the system.

In Fig. 3, the graph of the measured free damped acceleration $a_{i}^{e} \equiv a^{e}\left(t_{i}\right)$ is obtained at discrete time instances, $t_{i}$, increasing with a constant step $h^{e}=0.001 \mathrm{~s}$. It is seen that major experimental signal has a high frequency ripple, which fade together with the main dumped oscillations.

Fig. 4 shows a portion of absolute values of the measured acceleration $\left|a_{i}^{e}(t)\right|$ demonstrating closely the high frequency disturbance, which limits the proper measuring and getting accurate readings of the required parameters. These problems appear to be serious limitation when accurate results are needed for further applications and analysis. 


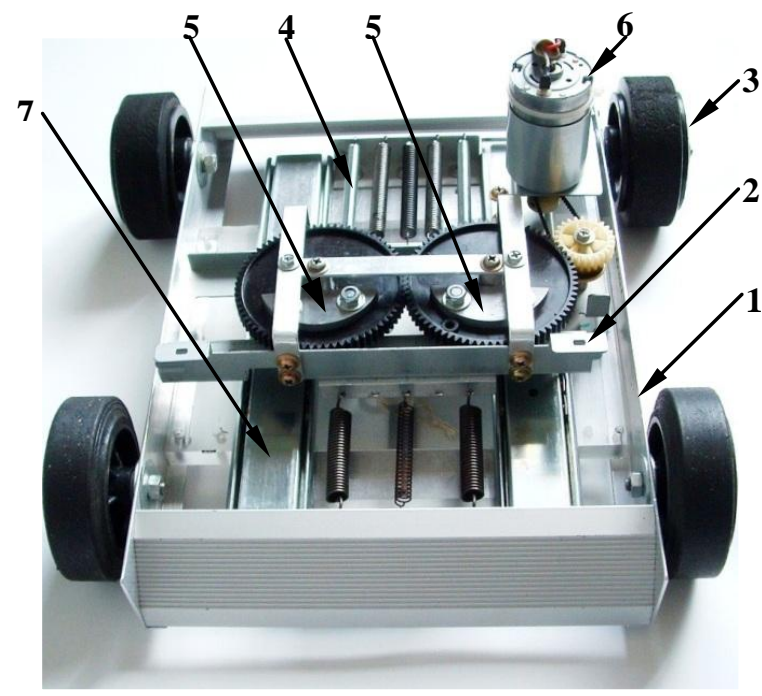

Fig. 1 shows the experimental prototype of the tested VibroBot: 1 - the chassis; 2 - shaker; 3 wheel with one-way bearing; 4 - springs; 5 rotating masses; 6 - motor, 7 - linear bearings

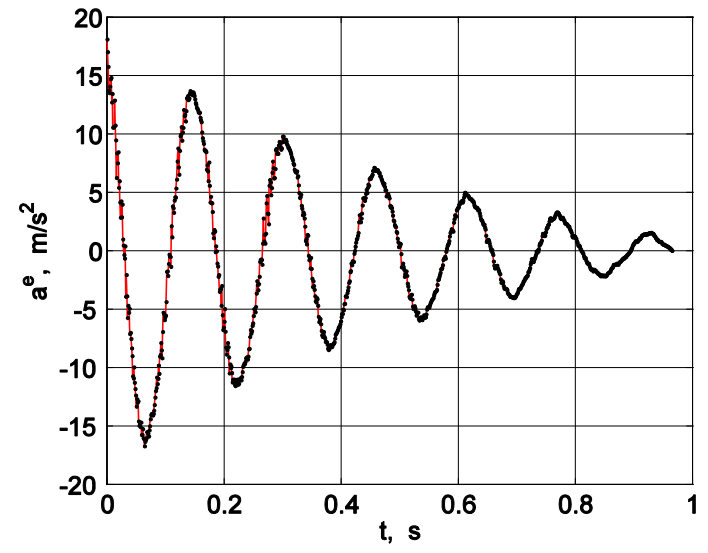

Fig. 3 depicts the recorded $(\cdot)$ acceleration $a^{e}(t)$ of free damped oscillations of shaker

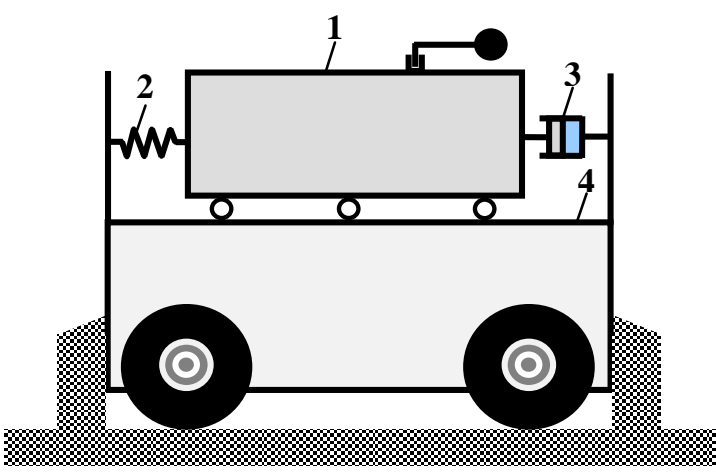

Fig. 2 illustrates the mechanical model of the robot: 1 - is the propulsion mechanism; 2 -equivalent spring; 3 -equivalent damper; 4 - provisionally fixed chassis

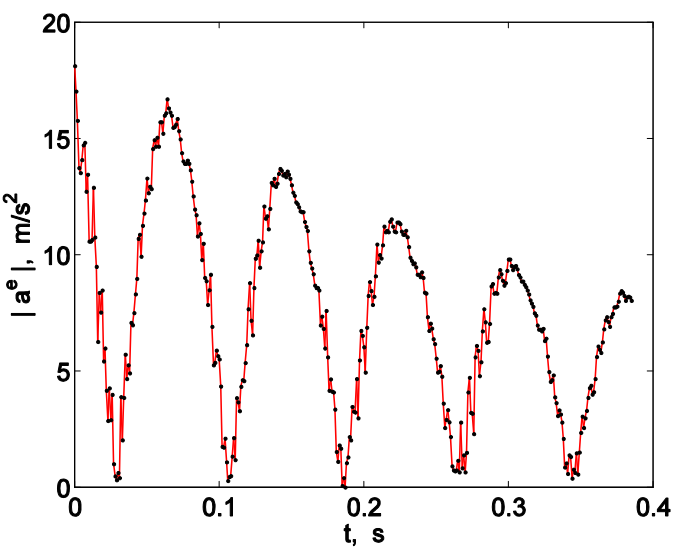

Fig. 4 Absolute values of the measured acceleration $a^{e}(t)$ within the time interval $t \in[0,0.38] \mathrm{s}$

\section{BI-CRITERIA FILTRATION}

A parametric identification of a model, simulating adequately the measured acceleration, can be significantly alleviated if the experimental data are smoothed out in advance. For this purpose the study [8] proposes a computational technology for bi-criterion polynomial smoothing using the least squares method to the discrete experimental data through the (S-G) filter of Savitzky-Golay [9].

For the realization of the smoothening process with the $(S-G)$ filter the function "sgolayfilt", which is a part of Signal Processing Toolbox of MATLAB system, is employed [10]. The choice of the governing parameters for the smoothing by this filter are the degree of the approximating polynomial - $n$ and the number of anchor points $-m$ is accomplished by Pareto-optimal [11] values of the controversial criteria "total absolute error" given by the equations:

$$
J=\sum_{i=1}^{n}\left|a_{i}^{e}-a_{i}^{g}\right|
$$

And the "integral smoothness"

$$
I=\int_{0}^{t_{f}}\left[\mathrm{~d}^{2} a^{g}(t) / \mathrm{d} t^{2}\right]^{2} \mathrm{~d} t
$$


where $a_{i}^{e} \equiv a^{e}\left(t_{i}\right)$ and $a_{i}{ }^{g} \equiv a^{g}\left(t_{i}\right)$ are the measured and smoothed values of the acceleration $a(t), i=1,2, \ldots, n^{e}$, at $t_{i} \in\left[0, t_{f}\right]$ respectively. Since $a_{i}{ }^{g}$ is a discrete function, the function (2) could be replaced by the approximation $\tilde{I}=\sum_{i=2}^{n_{e}-1}\left(a_{i+1}^{g}-2 a_{i}^{g}+a_{i-1}{ }^{g}\right)^{2}$. To determine the second time derivative - $\mathrm{d}^{2} a^{g}\left(t_{i}\right) / \mathrm{d} t^{2}$, the MATLAB-function "diff" [12] is employed.

In order to conclude the optimal in terms of the criteria (1) and (2) compromise values of adjustable parameters of the filter (S-G), a bi-criteria problem is formulated and solved:

$$
\begin{aligned}
& \operatorname{Pmin}_{\mathbf{u} \in \mathbf{D}} \mathbf{Q}(\mathbf{u}), \\
& \mathbf{Q}=[J(\mathbf{u}), \tilde{I}(\mathbf{u})], \quad \mathbf{u}=[n, m], \\
& \mathbf{D}=\left\{\mathbf{u} \in \mathbf{E}^{\mathbf{2}}: \mathbf{u}^{-} \leq \mathbf{u} \leq \mathbf{u}^{+}\right\},
\end{aligned}
$$

where "Pmin" is an operator for determining the global Pareto-minimal [11] compromise values of the vector criteria $\mathbf{Q}$, satisfying the condition that the vector $\mathbf{u}$ belongs to the possible domain $\mathbf{D}$, which is a rectangular area, defined by the boundary values $\mathbf{u}^{-}$and $\mathbf{u}^{+}$. Ranked, by the compromised effectiveness, Pareto subsets are determined by a method known as " $\mu$-selection" [13], [14] from the determined Pareto-optimal solutions. For the domain $\mathbf{D}$, having boundary values $\mathbf{u}^{-}=[2,31], \mathbf{u}^{+}=[10,71]$ the Salukvadze optimal solution of problem (3) is found as $\mathbf{u}^{\mathbf{S}}=[8,57], \mathbf{Q}^{\mathbf{S}}=[229.4,1.411]$ having the highest rank of a compromised effectiveness [15].

Fig. 5 illustrates the reached smoothness and closeness of the smoothed Salukvadze optimal acceleration $a^{g}$ as compared to the measured acceleration $a^{e}$.

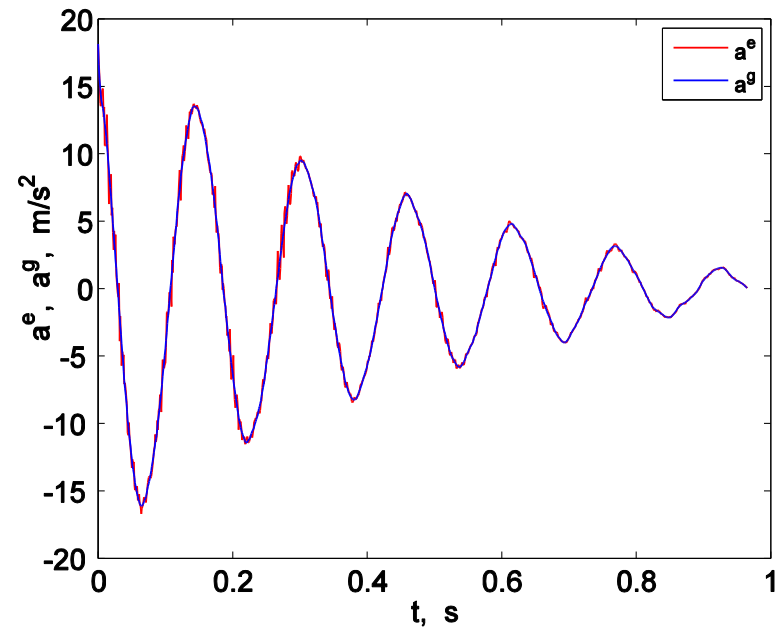

Fig. 5 The measured acceleration $a^{e}$ and the Salukvadze optimal smoothened acceleration $a^{g}$

\section{SIMULATION MODEL}

The justification of the choice of Mechano-mathematical model simulating reasonably the viscous damped free oscillations and serves for determination of the main dynamic characteristics of this process is possible through structural parametric identification. Now, to simplify the task this more general problem is fragmented into separate problems of parametric identification models with known structure.

In Table 1, four mechanical models are presented, having increasing complexity determined by the factor of "non-linearity", which can simulate free damped oscillations of a lumped mass, $m$. In the laws of elastic and dissipative forces $\left(F_{k}, F_{b}, F_{f}\right)$, the following parameters are used: $k$ and $c$ are the coefficients of linear and non-linear elasticity respectively; $b$ is the coefficient of viscous resistance; $f$ - is the coefficient of dry (Coulomb) friction; $x$ is the coordinate of the lumped mass position on axis $O x$ having a velocity - $v$ and $g-$ is the gravitational acceleration.

The most general of the models is the M4 model. The models M3, M2 and M1 can be considered as particular cases of M4. This is the reason to explore the model M4 first. It is described by a system of non-linear differential equations with initial conditions, presented in canonical form as follows:

$\mathrm{d} x(t) / \mathrm{d} t=v(t), \quad x(0)=s_{0}$

$$
\begin{aligned}
& \mathrm{d} v(t) / \mathrm{d} t=-\left\{\left[k+c x^{2}(t)\right] x(t)+b v(t)\right\} / m-f g \operatorname{sign}[v(t)], \\
& v(0)=0, \quad t \in \mathbf{I}_{t}=\left[0, t_{f}\right],
\end{aligned}
$$


where: $s_{0}=-0.012 \mathrm{~m}$ is the initial deviation from the equilibrium position of the lumped mass $m=0.120 \mathrm{~kg}$; $\operatorname{sign}(\cdot)-$ is the signum-function; $\mathrm{d} v(t) / \mathrm{d} t \equiv a^{c}(t)-$ the calculated acceleration from the model M4; $t_{f}=0.965 \mathrm{~s}-$ the termination time of the free damped oscillations.

With the help of the following substitutions:

$$
\begin{aligned}
& \mathbf{q}\left[\left(x-s_{0}\right), v\right], \mathbf{q}(0)=[0,0], \\
& \mathbf{u}=[k, c, b, f], \\
& \mathbf{\Phi}=\left[\mathrm{d}\left(x(t)-s_{0}\right) / \mathrm{d} t, \mathrm{~d} v(t) / \mathrm{d} t\right],
\end{aligned}
$$

the system of differential equations (4) is written in a compact form:

(6) $\quad \mathrm{d} \mathbf{q}(t) / \mathrm{d} t-\mathbf{\Phi}(t, \mathbf{q}(t), \mathbf{u})=\mathbf{0}, \quad \mathbf{q}(0)=\mathbf{0}, \quad t \in \mathbf{I}_{t}$.

In solving differential equations (4) with different values of the parameter vector $\mathbf{u}$, the step function $\operatorname{sign}(\cdot)$ may give rise to a numerical instability. To overcome this problem, it is approximated with the continuous analytical function $\operatorname{Sn}(v)=k_{1} \tan ^{-1}\left(k_{2} v\right)$, where: $k_{1}=0.6370253155$ is an experimentally determined

\begin{tabular}{|c|c|c|c|}
\hline Model & Force characteristics & Generalized mechanical model & \\
\hline M1 & $\begin{array}{l}F_{k}=-k x \\
F_{b}=-b v \\
F_{f}=0\end{array}$ & \multirow{4}{*}{ OO } & \\
\hline M2 & $\begin{array}{l}F_{k}=-\left(k+c x^{2}\right) x \\
F b=-b v \\
F_{f}=0\end{array}$ & & $m$ \\
\hline M3 & $\begin{array}{l}F_{k}=-k x \\
F_{b}=-b v \\
F_{f}=-f m g \operatorname{Sn}(v)\end{array}$ & & $x$ \\
\hline M4 & $\begin{array}{l}F_{k}=-\left(k+c x^{2}\right) x \\
F_{b}=-b v \\
F_{f}=-f m g \operatorname{Sn}(v)\end{array}$ & & \\
\hline
\end{tabular}
factor; $k_{2}=10^{3}$ - is a coefficient of the slope of the transitions between linear fragments.

Table 1 Mechano-mathematical models

For numerical integration of differential equations (4) with the function $\mathrm{Sn}(\cdot)$, the MATLAB program "ode113" having relative accuracy of $10^{-6}$ and absolute precision of $10^{-8}$ was used.

The results of numerical integration of the used solver must satisfy the requirement of comparability of the calculated and measured data, i.e. to generate a constant time step $h^{c}$, coinciding with the step $h^{g}$ of the smoothed acceleration.

\section{CRITERIA FOR ADEQUACY}

The adequacy of the computational model can be estimated from the proximity of the simulated to the measured data with the aid of local (for individually measured values) and global (for all values in the specified time interval) criteria.

In Fig. 6, the introduced local criteria of proximity are shown, which measure the closeness between the smoothed $a^{g}\left(t_{i}\right)$ and simulated $a^{c}\left(t_{i}\right)$ peak amplitudes of the absolute values of the acceleration $a(t)$ at the sampling instants $t_{i}$ in the range $\mathbf{I}_{t}$.

The differences of the maximum $\left(\Delta a_{i}^{+}\right)$and the minimum $\left(\Delta a_{j}^{-}\right)$absolute values of the accelerations and the corresponding instances of time, $\Delta t_{i}^{+}$and $\Delta t_{j}^{-}$, characterize locally the errors for the pole positioned points and indirectly - the degree of phase displacement. The amounts of these differences for the entire interval $t \in \mathbf{I}_{t}$ perform the role of a global criteria.

We introduce the following designations:

$$
\begin{aligned}
& a_{i}^{g+} \equiv a^{g+}\left(t_{i}^{g+}\right)=\sup _{t i \in \tau \alpha}\left\{a^{g}\left(t_{i}\right)\right\}, \\
& a_{i}^{c+} \equiv a^{c+}\left(t_{i}^{c+}\right)=\sup _{t i \in \tau \alpha}\left\{a^{c}\left(t_{i}\right)\right\}, \\
& \Delta a_{i}^{+}=a_{i}^{c+}-a_{i}^{e+}, \quad \Delta t_{i}^{+}=t_{i}^{c+}-t_{i}^{g+}, \quad i=1,2, \ldots, n^{+}, \\
& \tau_{\alpha}=\left[t_{\alpha}^{g+}, t_{\alpha+1}{ }^{g+}\right) \quad \alpha=1,2, \ldots, n_{\alpha}, \quad n_{\alpha}=t_{f} / n^{+}, \\
& a_{j}^{g-} \equiv a^{g-}\left(t_{j}^{g-}\right)=\inf _{t j \in \tau \beta}\left\{a_{2}^{g}\left(t_{j}\right)\right\}, \\
& a_{j}^{c-} \equiv a^{c-}\left(t_{j}^{c-}\right)=\inf _{t j \in \tau \beta}\left\{a^{c}\left(t_{j}\right)\right\}, \\
& \Delta a_{j}^{-}=a_{j}^{c-}-a_{j}^{g-}, \quad \Delta t_{j}^{-}=t_{j}^{c-}-t_{j}^{g-}, \quad j=1,2, \ldots, n^{-},
\end{aligned}
$$




$$
\begin{aligned}
& \tau_{\beta}=\left[t_{\beta}{ }^{g-}, t_{\beta+1}{ }^{g-}\right), \quad \beta=1,2, \ldots, n_{\beta}, \quad n_{\beta}=t_{f} / n^{-}, \\
& a_{\lambda^{g}}=a^{g}\left(t_{\lambda}\right), \quad \lambda=0,1, \ldots, n^{g}, \quad h^{g}=t_{f} / n^{g}, \\
& a_{l}^{c}=a^{c}\left(t_{l}\right), \quad l=0,1, \ldots, n^{c}, \quad h^{c}=t_{f} / n^{c}, \\
& \Delta a_{\lambda}=a_{\lambda}^{c}-a_{\lambda}^{g}, \\
& n_{a}=\min \left\{n^{g}, n^{c}\right\}, \quad n_{p}=\min \left\{n^{+}, n^{-}\right\} .
\end{aligned}
$$

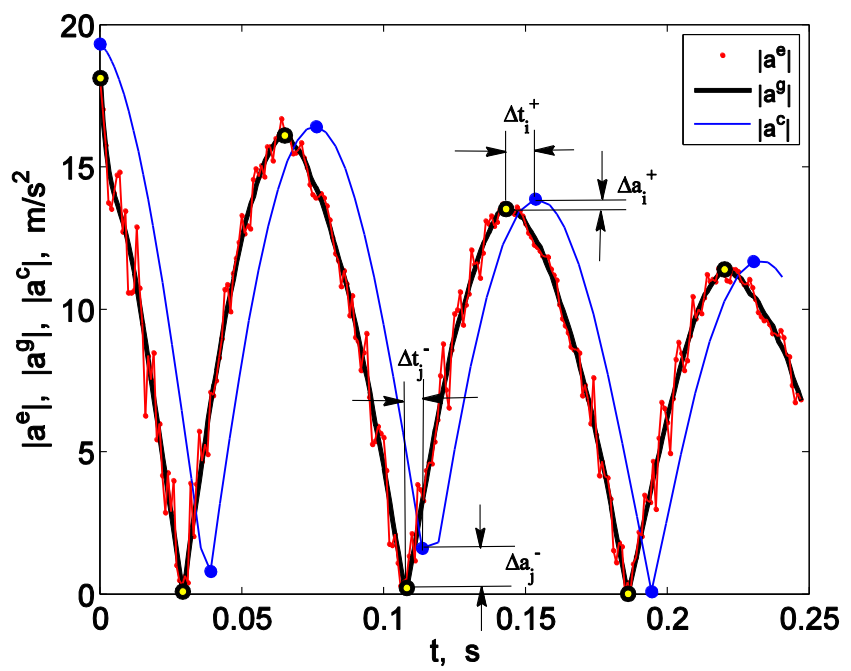

Fig. 6 illustrates the local criteria for proximity of the smoothed and the simulated absolute values of the acceleration $a(t)$

With the aid of designations (7) we formulate the following global criteria of proximity of amplitudes of the measured and calculated values of the acceleration $a(t)$ and the corresponding instances of time:

$$
\begin{aligned}
f_{1}= & \sum_{i=1}^{n_{a}}\left|\Delta a_{i}{ }^{+}\right|, \quad f_{2}=\sum_{i=1}^{n_{a}}\left|\Delta t_{i}{ }^{+}\right|, \\
f_{3} & =\sum_{j=1}^{n_{a}}\left|\Delta a_{j}^{-}\right|, \quad f_{4}=\sum_{j=1}^{n_{\lambda}}\left|\Delta t_{j}^{-}\right|, \\
f_{5} & \sum_{\lambda=1}^{n_{\lambda=1}}\left|\Delta a_{\lambda}\right| .
\end{aligned}
$$

Comparability between the measured and the calculated values of the criteria (8) is possible if the same time steps are used for the calculated and the measured data, respectively $\left(h^{c}=h^{g} \equiv h^{e}\right)$ as well as the following conditions should be satisfied

$$
n_{a}-n^{g}=0, \quad n_{p}-n^{n}=0
$$

where $n^{g}$ and $n^{n}$ are given numbers.

For the determination of the main characteristics of the measured free damped oscillations and the criteria of adequacy (8), it necessities finding the relevant extreme values of the discrete function $a^{g}\left(t_{i}\right)$, removing the pseudo-extreme values if they are available.

\section{MULTI-CRITERIA IDENTIFICATION PROBLEM}

The multi-criteria identification of the parametric vector $\mathbf{u}$ can be formulated as an optimization problem:

$$
\begin{aligned}
& \mathbf{u}^{*}=\arg \operatorname{Pmin}_{\mathbf{u} \in \mathbf{D}} \mathbf{f}(\mathbf{u}), \\
& \mathbf{f}=\left[f_{v}(\mathbf{u})\right], \quad v \in \mathbf{I}_{v}=\{1,2 \ldots 5\}, \\
& \mathbf{u}=[k, c, b, f], \\
& \mathbf{D}=\left\{\mathbf{u} \in \mathbf{E}^{4}: \mathbf{g}(\mathbf{u})=0, \mathbf{u} \in \mathbf{D}^{t} \cap \mathbf{\Pi}\right\}, \\
& \mathbf{D}^{t}=\left\{\mathbf{u} \in \mathbf{E}^{4}, \mathbf{q}(t) \in \mathbf{E}^{2}: \mathrm{d} \mathbf{q}(t) / \mathrm{d} t-\mathbf{\Phi}(t, \mathbf{q}(t), \mathbf{u})=\mathbf{0}, \mathbf{q}(0)=\mathbf{0}, t \in \mathbf{I}_{t}\right\}, \\
& \boldsymbol{\Pi}=\left\{\mathbf{u}^{-} \leq \mathbf{u} \leq \mathbf{u}^{+}\right\},
\end{aligned}
$$


where: "Pmin" is an operator for determining the global Pareto minimal compromise values of the criterion vector $\mathbf{f}$ defined by the equations (8); $\mathbf{g}(\mathbf{u})=\left[n_{a}-n^{g}, n_{p}-n^{n}\right]=\mathbf{0}$ - are the conditions (9) for comparability; $\mathbf{u}^{-}$ and $\mathbf{u}^{+}$are the specified boundary values of the searched vector $\mathbf{u}$. The boundaries of the domain $\mathbf{D}$ are determined by numerical experiment so that the Pareto optimal points $\mathbf{u}^{*}$ are internal to this region.

For any particular choice of the permissible parametric vector $\mathbf{u}$, the differential equations (6) are satisfied with desired accuracy in their numerical integration. That's why their role of differential constraints in the task (10) is limited to generation of calculated extended state $\left(x^{c}(t), v^{c}(t), a^{c}(t)\right)$ of the simulated mechanical system.

\section{OPTIMIZATION PROCEDURE}

The solution of the task (10) is based on the principle of coherent optimality of V. Pareto as suggested in [11]. The optimal solution $\left(\mathbf{u}^{*}, \mathbf{f}^{*}\right)$ consists of two sets of Pareto - multitudes $\mathbf{D}^{*}=\left\{\mathbf{u}^{*}: \mathbf{u}^{*}=\arg \operatorname{Pmin}{ }_{\mathbf{u} \in \mathbf{D}} \mathbf{f}(\mathbf{u})\right\}$ and $\mathbf{P}^{*}=\left\{\mathbf{f}^{*}: \mathbf{f}^{*}=\mathbf{f}\left(\mathbf{u}^{*}\right)\right\}$ with un-improving points $\mathbf{u}^{*} \in \mathbf{D}^{*}$ and $\mathbf{f}^{*} \in \mathbf{P}^{*}$. The choice of a compromised solution can be facilitated significantly if it is implemented by reasonably reduced subsets of $\mathbf{D}^{*}$ and $\mathbf{P}^{*}$. The task (10) is solved with the MATLAB program "psims" for multi-criteria parametric optimization by means of $\boldsymbol{\mu}$-selection procedure, documented in [14]. Optimization is carried out in two stages.

In Stage 1, approximate Pareto optimal sets $\mathbf{D}^{*}$ и $\mathbf{P}^{*}$ are determined with the PSI method (Parametric Space Investigation) intended for investigating multidimensional spaces through quasi-uniform signalling by means of Sobol's test points [16], [17].

In Stage 2, ranked by a compromise effectiveness Pareto subsets are selected with the aid of the minimum values $\mu_{k}^{*}, k \in \mathbf{I}_{k}=\{1,2,3\}$ of the components of vector criterion $\boldsymbol{\mu}=\left[\mu_{1}, \mu_{2}, \mu_{3}\right]$ chosen from the set $\mathbf{M}=\left\{\boldsymbol{\mu}\left(\mathbf{f}^{*}\right) \in \mathbf{E}^{3}: \mathbf{f}^{*} \in \mathbf{P}^{*}\right\}$. They correspond to the distances between three characteristic points: positive utopian point $\mathbf{f}^{U}$ with components of uncompromised minima of the particular criteria; the current compromise point $\mathbf{f}^{*}$ and its projection $\mathbf{f}^{U N}$ on the line $U N$, which joins both utopian points - the positive $\mathbf{f}^{U}$ and the negative $\mathbf{f}^{N}$ with components of the uncompromising maximums of the particular criteria. In general, the minimum distances $\mu_{k}{ }^{*}$ correspond to different Pareto optimal point's $\mathbf{f}^{*}$.

With the aid of the vector criterion $\boldsymbol{\mu}^{p}=\left[\mu_{1}{ }^{p}, \mu_{2}{ }^{p}, \mu_{3}{ }^{p}\right], p \in \mathbf{I}_{p}=\left\{1,2, \ldots, N_{P}\right\}$, for every Pareto optimal point $\mathbf{f}^{* p} \in \mathbf{P}^{*}$ the multiple $\mathbf{P} *$ is transformed into a point of the set $\mathbf{M}=\left\{\boldsymbol{\mu}^{p} \in \mathbf{E}^{3}: p \in \mathbf{I}_{p}\right\}$ in the 3-dimensional $\boldsymbol{\mu}$ space. All possible combinations of two criteria $\left\{\mu_{t}{ }^{p}, \mu_{h}{ }^{p}\right\}, t \neq h, t, h \in \mathbf{I}_{k}$ are investigated in the set $\mathbf{M}$. For each pair of criteria, subsets of Pareto optimal points $\mathbf{M}_{\rho}=\left\{\mu^{p} \in \mathbf{E}^{3}: \mu_{t}{ }^{p, t} \leq \mu_{t}^{p} \leq \mu_{t}{ }^{p, h}, h \neq t, t, h \in \mathbf{I}_{k}, p \in \mathbf{I}_{p}\right\} \subset \mathbf{M}$, $\rho \in \mathbf{I}_{\rho}=\{1,2, \ldots, 6\}$ are selected, where the minimizing point $\mu_{h}{ }^{* p, h}=\min _{p \in \mathbf{I} p}\left\{\mu_{h}{ }^{p}\right\}$ of a given criterion $\mu_{h}{ }^{p}$, $h \in \mathbf{I}_{k}$ is used as an upper limit in selection of another criterion $\mu_{t}^{p}, t \neq h, t \in \mathbf{I}_{k}$, and the minimum point $\mu_{t}^{* p, t}=\min _{p \in \mathbf{I} p}\left\{\mu_{t}^{p}\right\}$ of the criterion $\mu_{t}^{p}-$ is used as a lower limit. Every point selected in that way $\boldsymbol{\mu}^{\circ} \in \mathbf{M}_{\rho}$, $\rho \in \mathbf{I}_{\rho}$ and the corresponding point $\mathbf{f}^{\mathbf{o}} \in \mathbf{P}_{\boldsymbol{R}} * \subset \mathbf{P}^{*}$ receive as an individual estimator the number $R_{E}=\max \{\rho\}$, that defines their rank of compromise effectiveness. This number corresponds to the number $\rho$ of the subsets $\mathbf{M}_{\rho}$ in the incorporated set $\mathbf{M}_{R}=\left\{\cap_{\rho \in \mathbf{I} \rho} \mathbf{M}_{\rho}\right\}$ to which $\mu^{\mathbf{o}}=\boldsymbol{\mu}\left(\mathbf{f}^{\mathbf{o}}\right)$ belongs to.

The subset of $\mathbf{P}_{R}$ * with the highest rank $R_{E}=6$ usually contains only one point $\mathbf{f}^{S}=\mathbf{f}\left(\mathbf{u}^{S}\right)$, which corresponds to the Salukvadze optimal solution $\left(\mathbf{u}^{\mathbf{S}}=\arg \min _{\mathbf{u} \in \mathbf{D}} \mu_{3}(\mathbf{f}(\mathbf{u})), \mathbf{f}^{S}=\mathbf{f}_{\left(\mathbf{u}^{S}\right)}\right)$ [15] of the problem (10). This decision reveals the potential for steady bringing the particular criteria to their uncompromising optimal values under the assumption that they are equally-valued.The final compromise solution $\left(\mathbf{u}^{\#}, \mathbf{f}^{\#}\right)$ is chosen after analysis of the ranked Pareto-optimal subsets in $\mathbf{P}_{\boldsymbol{R}} *$.

First the Salukvadze optimal solution is analyzed. If it is assessed as unacceptable on the reached level of compromise by any of the particular criteria then the subsets of the $\mathbf{P}_{\boldsymbol{R}}{ }^{*}$ with a lower rank are consistently analyzed until a definite choice is made.

\section{RESULTS}

With the help of numerical experiment in a starting permissible parametric domain $\mathbf{\Pi}$, determined by the limits $\mathbf{u}^{-}=\left[2000,-10^{-5}, 5,0\right], \mathbf{u}^{+}=\left[2300,10^{-5}, 10,0.1\right]$ of the vector $\mathbf{u}$ in a growing numbers $N_{S} \in\left\{2^{8}, 2^{10}, 2^{12}\right\}$ of the Sobol's test points, a new subdomain $\boldsymbol{\Pi}$ is defined for which $\mathbf{u}^{-}=\left[2100,-10^{-5}, 5.5,0\right]$, $\mathbf{u}^{+}=\left[2200,-8(10)^{-4}, 6.5,0.008\right]$. This choice is made by the need to increase the effectiveness of examination. The newly discovered eligible domain $\mathbf{D}$ is examined with $N_{S}=2^{13} \equiv 8192$ Sobol's points.

In Stage 1 of the optimization procedure 418 Pareto optimal points $\mathbf{f}\left(\mathbf{u}^{*}\right)$ are defined. The utopian points $\mathbf{f}^{U}=[2.6725,0.03200,0.2779,0.03100,964.9], \mathbf{f}^{N}=[8.5922,0.06200,1.0621,0.05700,1415.4]$, specify in the space of attainable criteria, a line $U N$, which defines the direction of the agreed amendment of the particular criteria. The components of the ideal point $\mathbf{f}^{U}$ are the uncompromising minima $\mathbf{f}^{*}$ of the introduced criteria, $f_{v}, v \in \mathbf{I}_{v}$.

In Stage 2 a $\mu$-selection is carried out. For the model M4 six non-empty ranked Pareto subsets with rank $R_{E} \in\{6,5, \ldots, 1\}$ are determined. The solution $\left(\mathbf{u}^{S}, \mathbf{f}^{S}\right)$ with the highest ranking $R_{E}=6$ is the Salukvadze 
optimum, since it corresponds to the assumption of equal-valued particular criteria, the reached levels $f_{v}{ }^{S}, v \in \mathbf{I}_{v}$ of a compromise reveal the potential opportunity for their even reduction.

The Salukvadze optimal solutions of problem (10) for all the models listed in Table 1 are presented in Table 2. In the Figs. 7 to 9, the results in graphical form for the model M4 are displayed. The point with the highest rank $R_{E}=6$ is designated with the symbol ( $\square$ ) and points with rank $5,4, \ldots, 1$ with $(\bullet, \boldsymbol{\Delta}, \triangleright,\langle, \downarrow)-$ respectively.

Table 2 lists the Salukvadze optimum solutions $\left(\mathbf{u}^{\mathbf{S}}, \mathbf{f}^{\mathbf{S}}\right)$ of the parameters of mechanical system

\begin{tabular}{|c|c|c|c|c|c|c|c|c|c|}
\hline \multirow[t]{2}{*}{ Model } & \multicolumn{4}{|c|}{$\mathbf{u}^{S}$} & \multicolumn{5}{|c|}{$\mathbf{f}^{S}$} \\
\hline & $\begin{array}{c}k \\
\mathrm{~N} / \mathrm{m}\end{array}$ & $\begin{array}{c}c \\
\mathrm{~N} / \mathrm{m}^{3}\end{array}$ & $\begin{array}{c}b \\
\mathrm{Ns} / \mathrm{m}\end{array}$ & $\begin{array}{l}f \\
-\end{array}$ & $\begin{array}{c}f_{1} \\
\mathrm{~m} / \mathrm{s}^{2}\end{array}$ & $\begin{array}{l}f_{2} \\
\mathrm{~s}^{2}\end{array}$ & $\begin{array}{c}f_{3} \\
\mathrm{~m} / \mathrm{s}^{2}\end{array}$ & $\begin{array}{l}f_{4} \\
\mathrm{~s}^{2}\end{array}$ & $\begin{array}{c}f_{5} \\
\mathrm{~m} / \mathrm{s}^{2}\end{array}$ \\
\hline M1 & 2123 & 0 & 6.2149 & 0 & 4.1748 & 0.03900 & 0.3284 & 0.03400 & 1047.3 \\
\hline M2 & 2136 & -43218 & 6.2798 & 0 & 4.4170 & 0.03500 & 0.3700 & 0.03100 & 978.2 \\
\hline M3 & 2134 & 0 & 6.2156 & $5.6519(10)^{-4}$ & 4.3819 & 0.03500 & 0.3672 & 0.03200 & 973.16 \\
\hline M4 & 2137 & -83279 & 6.0094 & $1.7285(10)^{-4}$ & 3.6570 & 0.03600 & 0.4214 & 0.03300 & 1020.7 \\
\hline
\end{tabular}

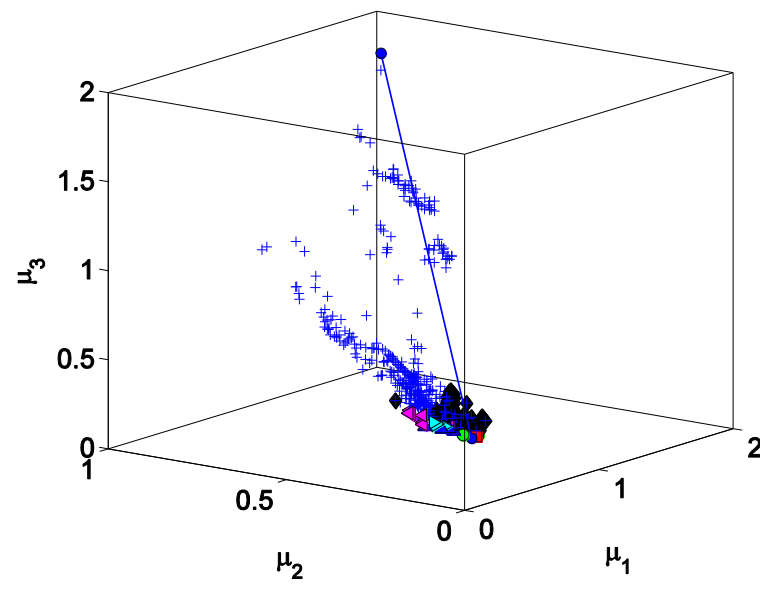

Fig. 7 shows Pareto optimal (+) and ranked points in the $\mu$ - space for the model M4

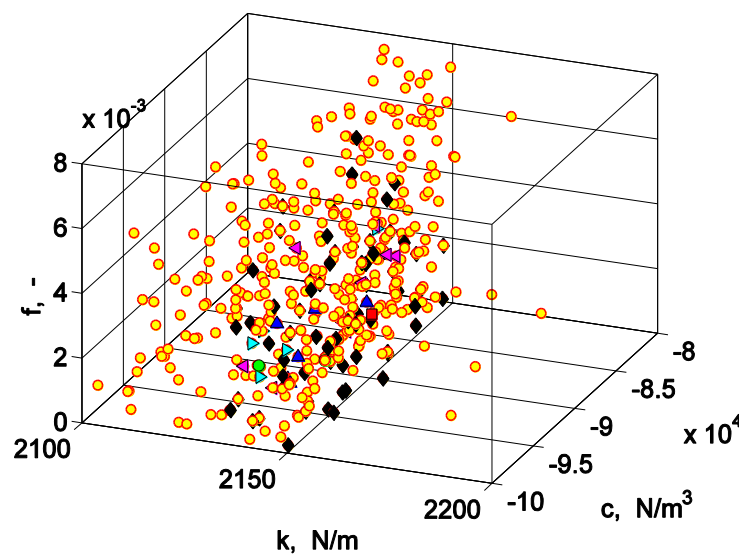

Fig. 9 Pareto optimal and ranked points in subsets $\mathbf{D}_{\mathbf{2}}=\left\{\mathrm{u}_{2} \in \mathrm{E}^{3}: g\left(\mathrm{u}_{2}\right)=0, \mathrm{u}_{2} \in \Pi_{2}\right\}, \quad \boldsymbol{\Pi}_{\mathbf{2}}=\left\{\mathrm{u}_{2}: \mathrm{u}_{2}{ }^{-}\right.$ $\left.\leq \mathrm{u}_{2} \leq \mathrm{u}_{2}{ }^{+}\right\}$, and $\mathbf{u}_{2}=[k, c, f]$ for the model M4

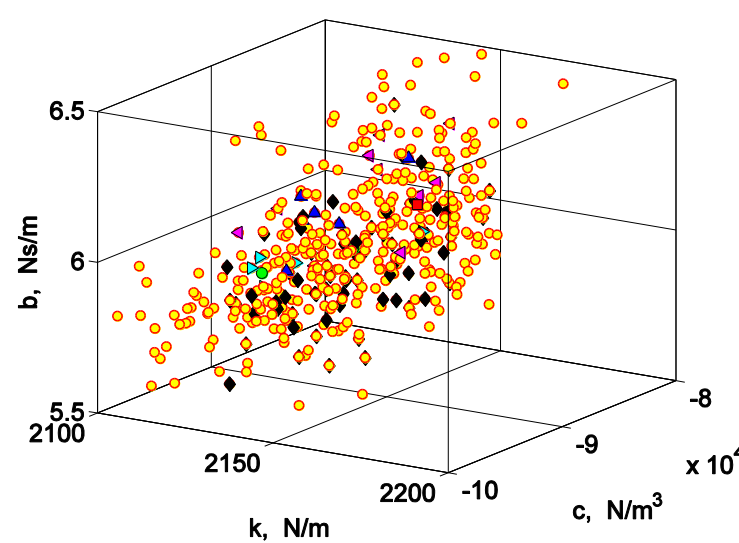

Fig. 8 clarifies Pareto optimal and ranked points in subsets $\mathbf{D}_{\mathbf{1}}=\left\{\mathrm{u}_{1} \in \mathrm{E}^{3}: g\left(\mathrm{u}_{1}\right)=0, \mathbf{u}_{\mathbf{1}} \in \Pi_{1}\right\}$, $\boldsymbol{\Pi}_{\mathbf{1}}=\left\{\mathrm{u}_{1}: \mathrm{u}_{1}^{-} \leq \mathrm{u}_{1} \leq \mathrm{u}_{1}^{+}\right\}$, and $\mathbf{u}_{\mathbf{1}}=[k, c, b]$ for the model M4

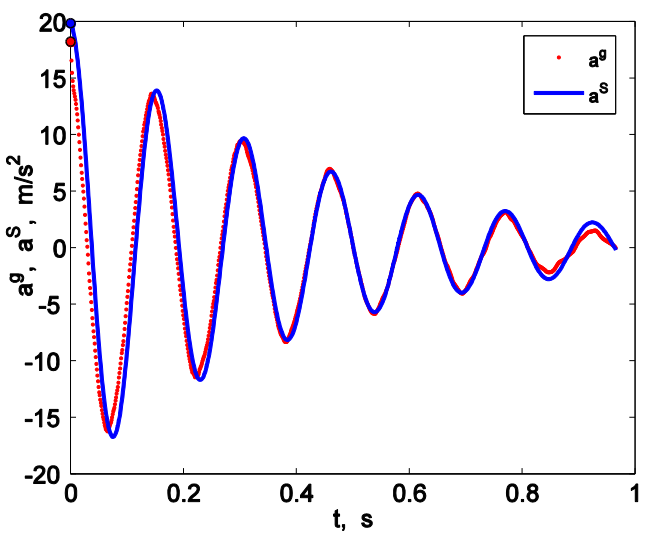

Fig. 10 displays the computed and smoothed $a^{g}$ acceleration as well as the Salukvadze optimal acceleration $a^{S}$ 
Fig. 10 illustrates the minor difference between the smoothed $\mathrm{a}^{\mathrm{g}}$ acceleration as well as the Salukvadze optimal acceleration $\mathrm{a}^{\mathrm{S}}$, indicating that the proposed procedure of simulation and smoothing is adequate and realistic. This also means that one could thrust the results obtained in this section and the process of smoothing of the experimental data is correct.

The analysis of the data of Table 2 shows that the model M4 is the dominant under the criterion $f_{1}$ over other models. It has similar values under criteria, $f_{2}, f_{3}, f_{4}$, and under criterion $f_{5}$ dominates only the M1 model. The established acceptable compromise effectiveness of the model M4 is a reason to choose it for a synonymous solution $\left(\mathbf{u}^{\#}, \mathbf{f}^{\#}\right)$ of the optimization problem (10).

In Fig. 10 the graphs of the measured smoothened acceleration $a^{g}(t)$ and the simulated by the model M4 of the Salukvadze optimum acceleration $a^{S}(t)$ are presented. It may be seen that the simulated graph is very close to the recorded and smoothed acceleration graph.

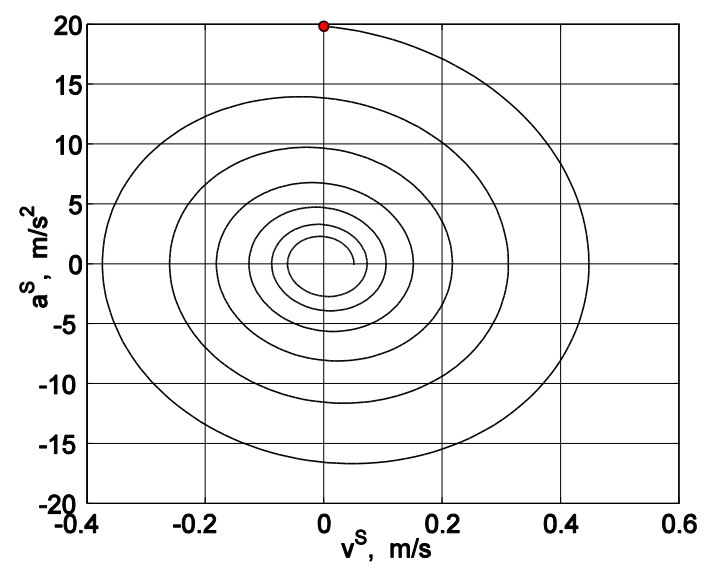

Fig. 11 illustrates the simulated acceleration $a^{S}$ as a function of the simulated velocity $v^{S}$ for the model M4

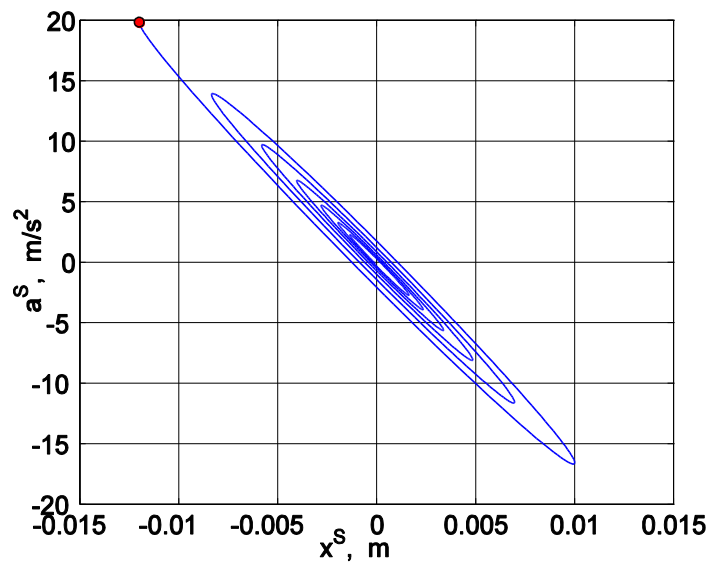

Fig. 12 demonstrates the simulated acceleration $a^{S}$ as a function of the simulated displacement $x^{S}$ for the model M4

The basic dynamic and kinematic characteristics simulated with the model M4 and the Salukvadze optimal values of the parameter vector $\mathbf{u}^{\mathbf{S}}$ are shown in Figs. 11-15. It is seen from these figures that the simulated process of damped oscillations such as simulated acceleration $a^{S}$, velocity $v^{S}$ and displacement $x^{S}$ is gradually decaying because of the available damping in the system. The degree of decay of these variables corresponds to the actual one measured in the study [8]. This suggests that the simulation procedure is correct and provide reasonable variations of these parameters.

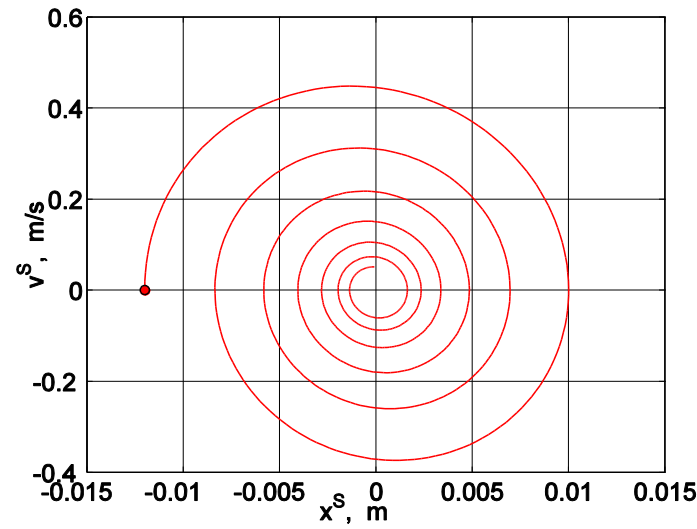

Fig. 13 displays the velocity $v^{S}$ as a function of the displacement $x^{S}$ for the model M4

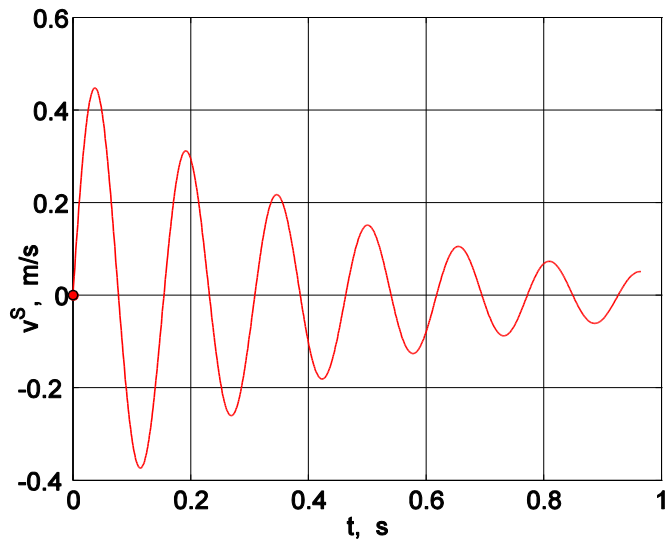

Fig. 14 shows the simulated velocity $v^{S}$ as a function of time for the model M4 


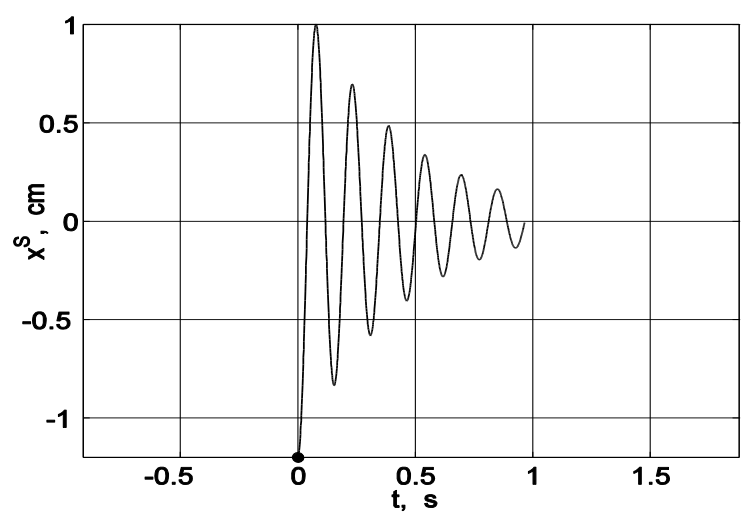

Fig. 15 presents the simulated displacement $x^{S}$ as a function of time for the model M4

\section{CONCLUSIONS}

The proposed approach for multi-criterion identification of the parameters of the VibroBot mechanical system allowed us to justify and build an adequate simulation model that describes realistically the robot dynamics. The existence of such a model is a prerequisite step for the mechanical improvement of this type of mobile systems at the stages of their design and experimental study.

We believe that the established mathematical model could also be very useful in assessing the dynamic behaviour of the newly designed and build Vibrobot capable of achieving not only a forward motion but also a backward motion, because it is also employing one-way rotating bearings. The design of the new robot has free rotating wheels and uses friction rollers with built in one-way rotating bearings. These rollers are actuated in such a way so as to come into friction contact with the freely rotating wheels of the robot. As a result a forward or backward motion of the robot is achieved accordingly.

\section{REFERENCES}

[1] Chernousko, F. Zimmermann, K. Bolotnik, S. Yatsun, I. Zeidis. Vibration-driven robots, Workshop on Adaptive and Intelligent Robots: Present and Future. Proceedings. The Institute for problem in mechanics RAS, Moscow, Vol. 1, 26-31, 2005.

[2] Jatsun, S.F., N.N. Bolotnik, K. Zimmerman, I. Zeidis. Modeling of motion of vibrating robots, 12-th IFToMM World Congress, Besançon, France, June 18-21, 2007.

[3] Jatsun, S., V. Dyshenko, A. Yatsun, and A. Malchikov. Modelling of robot's motion by use of vibration of internal masses. Proceedings of EUCOMES 08, the Second European Conference on Mechanism Science. M. Ceccarelli (Ed.) (Springer, New York: 262-270, 2009).

[4] Bolotnik, N., I. Zeidis, K. Zimmermann, S. Yatsun. Vibration-driven robots. 56th International Scientific Colloquium. Ilmenau University of Technology, 2011.

[5] Provatidis, C.G. Design of a propulsion cycle for endless sliding on friction ground using rotating masses. Universal Journal of Mechanical Engineering, Vol. 2, No. 2: 35-43, 2014.

[6] Loukanov, I.A. Inertial propulsion of a mobile robot. IOSR Journal of Mechanical and Civil Engineering, Vol. 12, No. 2, Ver. II: 23-33, 2015 a.

[7] Loukanov I.A., S.P. Stoyanov. Experimental determination of dynamic characteristics of a vibrationdriven robot. IOSR Journal of Mechanical and Civil Engineering, Vol. 12, No. 3: 62-73, $2015 \mathrm{~b}$.

[8] Vitliemov, V.G., I.V. Ivanov, I.A. Loukanov. Bi-criteria smoothing of data with Savitzky-Golay filter. Proceedings of University of Ruse, Vol. 54, Book 2, 2015, (in Bulg.).

[9] Gander, W., J. Hřebíček. Solving Problems in Scientific Computing Using Maple and MATLAB (Springer, Berlin, 2004).

[10] Signal Processing Toolbox ${ }^{\mathrm{TM}}$ User's Guide. (MathWorks, Inc., Natick, MA, 2015), http://www.mathworks.com/help/pdf_doc/signal/signal_tb.pdf

[11] Ehrgott, M. Multicriteria Optimization (Springer, Berlin, 2005).

[12] Symbolic Math Toolbox TM User's Guide. (MathWorks, Inc., Natick, MA, 2015), https://www.mathworks.com/help/pdf_doc/symbolic/symbolic_tb.pdf

[13] Cheshankov, B.I., I.V. Ivanov, V.G. Vitliemov, P.A. Koev. PSI-method multi-criteria optimization contracting the set of trade-off solutions. 15th International Conference on Systems Science, Wroclaw, Poland, Vol. 1: 281-288, 2004. 
[14] Jordanov, J.T, V.G. Vitliemov. Optimization with MATLAB. Pragmatic Approach (University Press „Angel Kanchev”, Ruse, 2013 in Bulg.). Also available on the following website: http://ecet.ecs.uni-ruse.bg/else/subjects/_index.php?cid=2130211020910970

[15] Salukvadze, M.E. Vector-Valued Optimization Problems in Optimal Control Theory (Academic Press, New York, 1979).

[16] Statnikov, R.B., J.B. Matusov. Multicriteria Analysis in Engineering. (Kluwer Academic Publishers, Dordrecht, 2002).

[17] Statnikov, R.B., A.R. Statnikov. The Parameter Space Investigation Method Toolkit. (Artech House, Boston/London, 2011).

[18] Loukanov I.A. Application of Inertia Forces for Generating Unidirectional Motion, Scientific Conference organized by the University of Ruse "Angel Kanchev" and the Union of Scientists in Ruse, 2015. Also posted on the website: http://conf.uni-ruse.bg/bg/?cmd=dPage\&pid=proceedings 